\title{
Armazenamento em baterias no contexto da expansão da fonte eólica no Brasil
}

\author{
Daniel C. de H. Cavalcanti Filho ${ }^{1,2}$ (D) orcid.org/0000-0001-9236-8563
}

\section{Pedro André C. Rosas ${ }^{2,3}$ orcid.org/0000-0001-9680-7228}

\footnotetext{
${ }^{1}$ Escola Politécnica de Pernambuco, Universidade de Pernambuco, Recife, Brasil,

2 Pós-graduação em Energias Solar e Eólica, Escola Politécnica de Pernambuco, Pernambuco, Brasil,

3 Universidade Federal de Pernambuco, Recife, Pernambuco, Brasil.

E-mail do autor principal: Daniel C. de H. Cavalcanti Filho daniel.cavalcanti.f@gmail.com.br
}

\section{Resumo}

As tecnologias de armazenamento de energia estão sendo consideradas no Brasil e no mundo como solução de vários problemas dos sistemas elétricos relacionados com a inserção de grandes blocos de energia eólica, tornando os sistemas mais seguros e confiáveis. O número de parques eólicos tem crescido muito nos últimos anos, inserindo uma produção estocástica na rede elétrica. $O$ objetivo deste artigo é avaliar de que maneira as baterias podem ser utilizadas a fim de dar suporte ao sistema elétrico. Para tanto, foram estudados monografias, artigos e dados de órgãos públicos nacionais e internacionais a fim de buscar diretrizes de forma a avaliar a inserção de sistemas de armazenamento. Como consequência, é observado que o país tem a se beneficiar dessa nova tecnologia, mas precisa de cautela quanto a quantidade de sistemas de armazenamento a ser construído e seu impacto na economia.

Palavras-Chave: Sistemas de armazenamento de energia; Fonte eólica; Energias renováveis.

\begin{abstract}
Battery storage technologies are being considered in Brazil and around the world as a problem solver in electric systems related to the insertion of great amount of wind power, making them safer and more reliable. The quantity of wind farms has been growing strong in the last years and it is adding a stochastic production into the electric grid. The main objective of this paper is to evaluate how batteries can be utilized in order to support the electric system. Therefore, we studied national and international monographies, articles and public agencies' data to obtain guidelines to evaluate the insertion of storage systems. As consequence, it is observed that the country has much to benefit from this new technology, but it needs caution concerning the amount of storage systems to be raised and its impact in the national economy.
\end{abstract}

Key-words: Battery storage systems; Wind energy; Renewable energy. 


\section{Introdução}

A energia eólica está em forte expansão no Brasil pois é uma fonte renovável e de grande potencial graças à geografia brasileira. Porém sua operação é de difícil determinação, pois a energia gerada pode coincidir com congestionamento no sistema, ou seja, a entrega de energia que um parque eólico pode fornecer ao sistema elétrico depende da disponibilidade das linhas de transmissão. Seu uso em conjunto com sistemas de armazenamento será de grande auxílio à otimização do sistema de potência, melhorando a garantia de qualidade do fornecimento de energia e o barateamento do produto energia elétrica.

A perspectiva é que a exploração da fonte eólica aumente nos próximos anos devido aos incentivos de financiamento, necessidade de atendimento à carga futura, amadurecimento da tecnologia e sua aceitação pela população devido a sua característica de ser ambientalmente amigável.

\section{Eólicas e armazenamento no Sistema Interligado Nacional (SIN)}

A fonte eólica possui a característica de ter menor custo, porém tem baixa capacidade de entrega de potência. Sua energia entregue varia bastante ao longo do dia e é dependente das condições de vento, portanto sua operação é caracterizada como intermitente. Um outro problema é que a energia gerada pelos parques eólicos pode nem sempre ser comportada pela atual estrutura de rede elétrica, sendo necessário complementar a energia demandada utilizando usinas mais caras, contrariando a ideia de economia que o despacho por ordem de mérito emprega, ou direcionar energia de outro subsistema, o que torna-se complicado pois a variação de energia entregue pelas eólicas pode ser intensa em um curto período de tempo, requerendo sistemas de controle ágeis e altamente confiáveis [1].

o Sistema Interligado Nacional (SIN) é extremamente complexo e conecta todas as regiões do Brasil. A larga aplicação de usinas eólicas no sistema elétrico altera suas características e geram novas preocupações que precisam ser antecipadas e solucionadas para a operação sadia e segura do SIN.
Para aproveitar a energia eólica mais eficientemente é possível utilizar Sistemas de Armazenamento de Energia (SAE). Sua atuação consiste em armazenar energia proveniente de usinas eólicas e de outras fontes renováveis, como hidrelétrica e solar, durante os gargalos na rede de transmissão, diminuindo o despacho descentralizado de usinas caras como as de termelétricas. A energia armazenada passa a ser liberada à rede quando as linhas estiverem mais uma vez disponíveis. Assim é possível garantir o fornecimento de energia limpa e a baixo custo por mais tempo, evitando os altos custos de fontes poluentes.

Um empecilho para aplicações de sistemas com bateria é a escassez de projetos e necessidade amadurecimento da tecnologia, engenharia, manutenção e adequação ao mercado de energia [1, $2,3,4,5]$.

\subsection{Considerações para o Brasil}

Estima-se que, no Brasil, o recurso de maior expansão será o de energia eólica. Entre 2023 e 2027 deverá ser acrescido $2.000 \mathrm{MW}$ ao ano de capacidade eólica no SIN, como pode ser visto na Tabela 1 [1]. Tendo em vista essa expansão considerável, a ANEEL, em 2017, aprovou 23 propostas de Pesquisa e Desenvolvimento (P\&D) sobre armazenamento de energia para atender a demanda verificada no PDE 2027 [6].

Tabela 1: Potência instalada a ser acrescida, em megawatts (MW), da fonte eólica, de usinas termoelétricas (UTE) e de sistemas de armazenamento de energia (SAE) entre os anos de 2022 e 2027.

\begin{tabular}{l|l|l}
\hline \multicolumn{1}{c|}{ Fontes } & \multicolumn{1}{|c|}{2022} & \multicolumn{1}{c}{2027} \\
\hline UTE + SAE & 204 & 13.142 \\
\hline Eólica & 0 & 10.000 \\
\hline
\end{tabular}

Fonte: Adaptado de MME (2017, p. 64).

Portanto, para o crescimento saudável e seguro do SIN, os sistemas de armazenamento e as usinas termoelétricas devem crescer de maneira a auxiliar o aumento da fonte eólica. A bateria utilizada em SAE é uma tecnologia considerada importante no auxílio da expansão das energias renováveis por permitirem melhor gerenciamento das linhas de transmissão e do atendimento da demanda de energia [7]. 
No contexto dos agentes impactados pelo aumento significativo de SAE no futuro, se faz necessário atentar para: os operadores da rede, os proprietários dos SAE e os consumidores finais. Para atendê-los, os SAE precisam de incentivos financeiros, tributários, regulatório, de contratação, de capacitação tecnológica e de mão de obra [1, 8].

Hoje não existem aspectos regulatórios que tratam sobre tecnologias de armazenamento. Portanto este artigo faz referência ao manual do Electric Power Research Institute (EPRI) a fim de compartilhar os estudos realizados nessa área para nacionalização dos conceitos abordados.

\section{Aplicações e tecnologias dos SAE}

As várias tecnologias do SAE têm a capacidade de tornar o sistema, como um todo, mais eficiente e aberto às novas plantas eólicas em planejamento e construção.

\subsection{Controle de frequência e tensão}

Variação de frequência e tensão são indesejáveis ao sistema elétrico pois podem danificar aparelhos eletrônicos e máquinas. Para esta aplicação é recomendado o uso de baterias com capacidade de vida longa e alta velocidade de reação a fim de acompanhar as flutuações que o sistema está sujeito e torná-lo mais estável. O tempo de descarregamento para este tipo de aplicação é de até uma hora $[2,8]$.

Baterias indicadas para essas aplicações são as de lítio, chumbo-ácido, níquel-cádmio e níquel-hidreto metálico [2].

\subsection{Reserva de energia}

A energia elétrica pode ser armazenada em períodos de congestionamento das linhas de transmissão e fornecida posteriormente [9].

A Figura 1.a ilustra uma usina eólica $(E)$, um sistema de armazenamento de energia ( $S A E$ ), uma fonte hidrelétrica $(G)$ e uma fonte térmica $(T)$. Quando a potência gerada $\left(P_{G}\right)$ é maior do que a potência máxima permitida pela linha de transmissão $\left(P_{\text {máx }}\right)$ a energia excedente é direcionada ao SAE.
Uma vez que a linha de transmissão está mais uma vez disponível, o SAE fornece a energia limpa previamente armazenada ao sistema, conforme Figura 1.b.

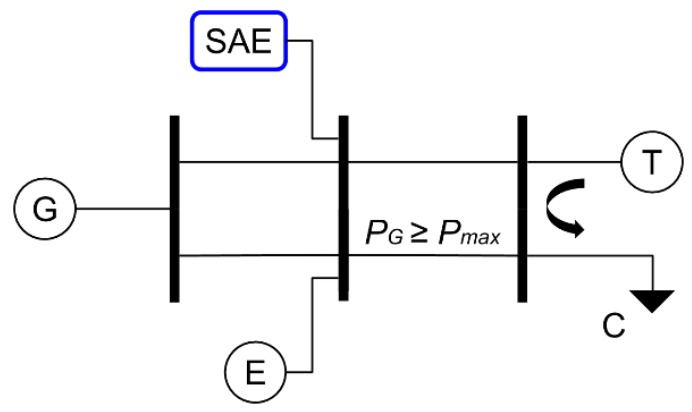

(a)

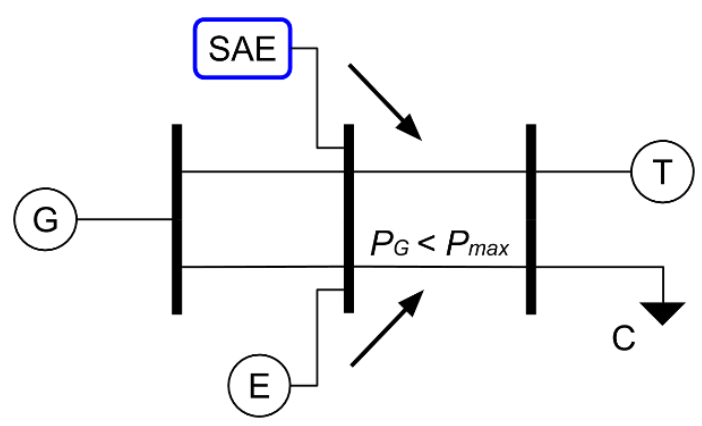

(b)

Figura 1: Sistema de três barramentos: (a) quando a potência gerada $\left(P_{G}\right)$ pelas fontes menos custosas é maior que a potência máxima permitida pela linha de transmissão $\left(P_{\max }\right)$ a demanda $(C)$ é atendida pela complementação de usinas térmicas $(T)$ e a energia em excesso é armazenada no SAE. (b) Uma vez que a linha de transmissão está disponível novamente, o SAE injeta a energia armazenada na rede, assim reduzindo as perdas da energia eólica que não pôde ser transmitida devido ao congestionamento na rede elétrica.

Fonte: Adaptado de Vargas, Bustus-Turu e Larraín (2014).

Essa é uma aplicação importante pois as baterias podem armazenar energia de baixo custo proveniente de usinas eólicas, solares, biomassa e nucleares a fim de fornecê-las ao SIN para auxiliar no atendimento à demanda. Mitigando a volatilidade dos preços ao consumidor final $[2,3,8]$.

Quanto maior o tempo que uma bateria pode fornecer energia à rede elétrica, menores são os cortes na transmissão de energia, portanto baterias de descarga lenta devem ser aplicadas, como as de lítio e chumbo-ácido. Porém não é recomendável que altos investimentos em vários sistemas de baterias 
com essa característica, uma vez que os ganhos aos empreendedores diminuem e o custo se eleva [2].

É necessário avaliar o quanto de armazenamento deverá ser inserido no sistema a fim de obter melhor desempenho do SIN, a um custo adequado ao consumidor, e tornando o empreendedorismo atrativo nessa área [10].

Baterias de lítio, chumbo-ácido, níquel-cádmio e níquel-hidreto metálico são indicadas para reservas de energia. Observando que as de lítio são mais caras do que as de chumbo ácido, porém essas possuem menor custo e o menor tempo de vida útil, sendo recomendadas para pequenos desenvolvimentos iniciais no país [2].

\subsection{Aumento da inércia da rede}

A rápida resposta dos SAE para garantir o atendimento à demanda torna o SIN mais resistente a variações de geração, assim, tornando a rede mais estável e segura aos consumidores e indústria [8].

Esse comportamento terá impacto direto no sistema de reserva girante no sistema elétrico, possibilitando uma segurança maior em relação a operação, qualidade e, em último caso, redução do custo operacional do sistema elétrico.

\subsection{Serviço de black start}

Black start é um serviço onde, após ocorrido desligamento total ou parcial de um sistema elétrico, ele é capaz de auxiliar no religamento desse sistema. Logo, um SEA pode atuar no lugar de um sistema ancilar de black start para religar o sistema em caso de desligamento [8].

Uma das principais aplicações que está sendo estudada pelo mundo é a redução dos cortes de energias renováveis devido à intermitência dessas fontes e o limite de transmissão da rede. A criação de modelos e aprimoramento da tecnologia pode tornar o armazenamento em baterias viável, seguro e importante para o atendimento à demanda crescente.
É possível observar, portanto, impactos no gerenciamento de energia e no mercado financeiro implicados pelas tecnologias de armazenamento de energia.

\section{Implementação de SAE no Brasil}

Se faz necessário avaliar a construção de novas usinas eólicas em conjunto com novas linhas de transmissão e/ou bancos de baterias a fim de decidir sobre a melhor alternativa para elaboração do plano de expansão elétrica. Deverá ser papel do Ministério de Minas e Energia (MME), Empresa de Pesquisa Energética (EPE) e Operador Nacional do Sistema Elétrico (ONS) estudar o local onde é necessário ter um SAE para fortalecer o sistema. As tecnologias de armazenamento poderão ser localizadas onde a construção de novas linhas de transmissão não é possível.

De acordo com pesquisadores membros do IEEE, o número de contingências no sistema elétrico diminui quando tecnologia de armazenamento é empregada juntamente com a expansão da rede desde a fase de planejamento, graças à sua capacidade de resposta em frequência. $O$ armazenamento possui valor não somente ambiental, mas também econômico para os interesses do país e da indústria, servindo como um serviço ancilar e reduzindo investimentos em linhas de transmissão [10].

O estudo do Ministério de Minas e Energia indica que o Nordeste terá $80 \%$ dos empreendimentos eólicos destinados, enquanto a região Sul terá $20 \%$, pois essas duas regiões possuem os melhores indicativos para aproveitamento de energia eólica no país. Ambas terão que estar preparadas para aceitar o aumento de parques eólicos para seu melhor aproveitamento [1].

\subsection{Armazenamento em sistemas isolados}

O Brasil possui diversos sistemas isolados na região Norte do país, a tecnologia de armazenamento poderá servir esses sistemas pois diminuiria os custos com geração de óleo diesel ao ser implementada em conjunto com fontes renováveis [3]. 


\subsection{Custo benefício de SAE}

O SIN tem dimensões continentais e a escolha do local ideal para a implementação de um SAE é de extrema importância para sua viabilidade econômica e operacional. Barramentos que conectam diversas
Analisando a Figura 2 percebe-se que os parques eólicos estão concentrados nos subsistemas Nordeste e Sul, estando também presentes em menor escala no subsistema Sudeste/Centro-Oeste. Neles estão localizadas as linhas de transmissão que poderão sofrer congestionamento com o aumento de usinas eólicas. E pode-se chegar à conclusão de que SAE

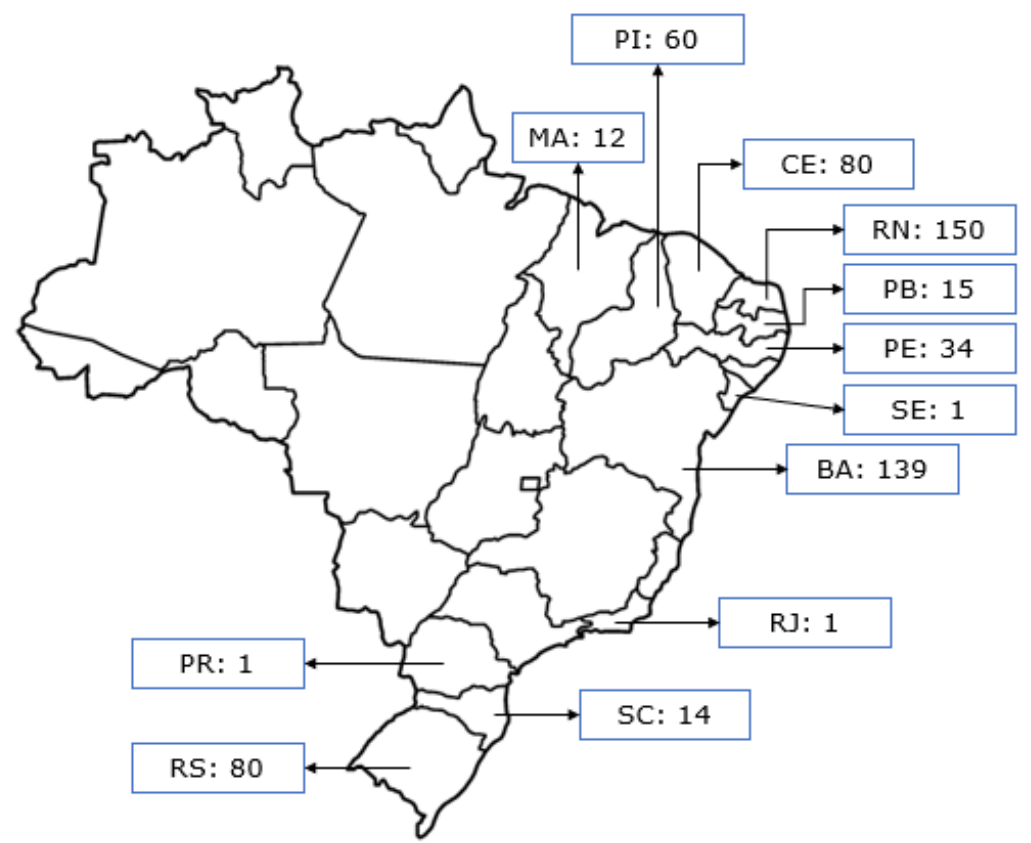

Figura 2: Mapa dos empreendimentos de geração de energia eólica no Brasil em 2019.

Fonte: adaptado de ABEEólica (2019).

fontes renováveis ou que são pontos de interconexão entre regiões que podem sofrer com congestionamento são preferência para servirem de conexão às SAE. Deve-se, porém, evitar a instalação de mais de um sistema de armazenamento de alta capacidade em barramentos próximos, uma vez que, para um local específico do sistema, os dois sistemas irão "competir" um com o outro, operando abaixo de capacidade nominal de armazenamento e, consequentemente, entregando menos energia ao SIN. Mais baterias conectadas ao sistema não significa mais benefícios para o mesmo, nem para os empreendimentos de baterias [4].

Para garantir que a quantidade ideal de armazenamento esteja disponível ao SIN e em quais barramentos eles serão conectados, a ONS e a EPE deverão fornecer quais são esses pontos a fim de dar aos empreendedores a capacidade de analisar seus investimentos de maneira adequada [12]. deverão ser instalados nos pontos dos subsistemas Nordeste, Sudeste-Centro-Oeste e Sul [13].

No Brasil, o preço da conta de energia está caro, pois a quantidade de energia eólica inserida não está sendo suficiente para atender a demanda de maneira continuada por causa de seu fator intermitente. Esse cenário atual torna o uso de SAE mais rentável, de acordo com estudo de [4].

\section{Desafios técnicos}

Se faz necessário quantificar uso de baterias em terra e seus desafios [2]. Estudos realizados pelo EPRI procuram dimensionar essas questões e antecipar problemas para conceber soluções para os SAE [14].

Os desafios relevantes associados a sistemas de armazenamento e geração eólica são:

- Quantificação do armazenamento; 
- A correta escolha do tipo de armazenamento; e

- A localização do mesmo.

\subsection{Mercado de energia}

A Câmara de Comercialização de Energia Elétrica (CCEE) precisa preparar as regras dos leilões de armazenamento de energia com o propósito de acolher esses empreendimentos da forma mais adequada possível ao mercado de energia elétrica. Baterias não geram energia, sua remuneração deverá ser baseada na energia que elas fornecem ao sistema, com o intuito de não perder a geração barata e limpa de fontes renováveis. Além das outras aplicações apresentadas neste trabalho.

O EPRI sugere que seja feita uma etapa eliminatória para o leilão, onde os empreendedores deverão ser aprovados nos seguintes quesitos [14]:

- Finanças sólidas;

- Ausência de conflitos de interesse;

- Ausência de antecedentes legais; e

- Ética de compromisso legal perante a CCEE.

É preciso comprovar que o local da instalação e as características técnicas do sistema de armazenamento de baterias irão servir ao bem do sistema e aos consumidores. Informações sobre performance, operação, tempo de vida útil e especificações de programação são importantes para assegurar que o empreendimento possui fundações sólidas.

Restrições de investimentos deverão ser implementadas para o crescimento sadio da rede elétrica, pois deve-se limitar a quantidade de empreendimentos e expansão de tecnologias de armazenamento para verificar sua viabilidade econômica [15].

O Brasil não possui um modelo oficial de suporte à inserção de sistemas de armazenamento para acompanhar a expansão da fonte eólica, porém existe estudo de modelagem realizado no programa de mestrado da Universidade do Rio de Janeiro, que serve como passo inicial a inserção da tecnologia de armazenamento de baterias [16].

Os modelos existentes de modelagem de sistemas de baterias, como o Regional Energy Deployment System (ReEDS), pertencente aos Estados Unidos, ainda são incompletos em relação a verificar oportunidades de negócio para favorecer expansão ou o controle de frequência da rede [12].

A economia que envolve a estruturação, modelos de negócio e regulações de SAE ainda precisa evoluir para a expansão dessa tecnologia [17].

\subsection{Evolução da tecnologia}

De acordo com o documentário Em Busca da Superbateria, do programa de televisão Nova, existem projetos de pesquisa e desenvolvimento para fabricação de baterias que utilizam eletrólito de plástico ao invés de fluido inflamável [18]. O interesse nesse tipo de projeto reside em tornar carros elétricos e aparelhos móveis mais seguros. Mas esse tipo de tecnologia também pode ser empregado em baterias em terra, pois são baterias mais leves, de mais fácil manutenção e mais seguras para o homem e o meio ambiente [18].

\subsection{Custos}

A eficiência dos sistemas de armazenamento está atrelada ao seu custo capital. É importante avaliar se sistemas de alto custo tem melhor custo benefício do que sistemas de baixo custo.

Numa projeção de 20 anos o custo capital dos SAE de baixo custo deve ter uma diminuição mais drástica em relação as de médio e alto custo conforme Figura 3, tornando esses sistemas mais acessíveis [12].

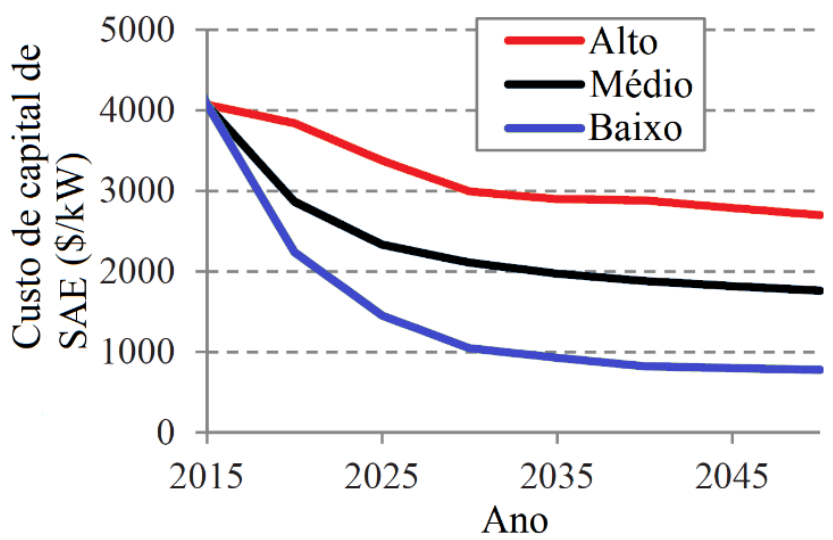

Figura 3: Custo capital de bateria de lítio com trajetórias de baixo, médio e alto custo para 8 horas de capacidade de entrega de energia em $\$ / \mathrm{KW}$.

Fonte: adaptado de Cole et al. (2016). 
Em relação a operação e manutenção, o alto preço das baterias também é um impedimento para o desenvolvimento da tecnologia atualmente. Espera-se que os custos de operação e manutenção de baterias de alto custo se mantenha elevado até 2050. Enquanto as de médio e baixo custo devem cair cerca de $50 \%$ e $66 \%$, respectivamente [12].

O estudo conduzido no laboratório de energia renovável dos Estados Unidos da América indica que as baterias de baixo custo devem liderar o mercado pela sua facilidade e retorno financeiro, seguidas pelas baterias de alto custo. Porém as baterias de alto custo possuem capacidade muito maior de energia de reserva para o sistema e evitar congestionamentos nas linhas de transmissão. Com o aumento esperado de usinas eólicas, espera-se um aumento na mesma proporção de $\mathrm{SAE}$, portanto é preciso observar que baterias de alto e médio custo possuem mais capacidade de reservar energia e servir ao SIN do que baterias de baixo custo [12].

\section{Conclusões}

Sistemas de armazenamento de energia mostramse uma boa solução para atenuar problemas de corte de transmissão energia devido a saturação da rede. Seu sucesso é importante para a expansão de fontes renováveis como a eólica, e manutenção da alta parcela de energia limpa que a matriz energética brasileira possui.

Para a pesquisa e desenvolvimento é aconselhável aplicação variada de tecnologias de armazenamento, como as de chumbo-ácido (baixo custo) e lítio (alto custo). Para tornar possível a coleta de informações pelos órgãos do governo, sistemas mais simples e variados deverão servir para análise econômica, de O\&M e de mercado.

Investimentos na evolução em tecnologia de baterias devem ser feitos para tornar esses empreendimentos atrativos aos financiadores e empresários. E estudos devem verificar se esses empreendimentos realmente irão introduzir preços menores de conta de energia a fim de favorecer a indústria a investir no Brasil.

\section{Referências}

[1] MINISTÉRIO DE MINAS E ENERGIA. Plano decenal de expansão de energia 2027.
Brasília, DF, 2017. 345 p.

[2] PARFOMAK, P. W. Energy storage for power grids and electric transportation: A technology assessment. In: LIBRARY OF CONGRESS, CONGRESSIONAL RESEARCH

SERVICE. [S.I.], 2012.

[3] LEITE, N. d. C.; DELGADO, M. A. d. P.; HAGE, F. A. S. Os desafios do armazenamento de energia no setor elétrico. Revista Boletim da Conjuntura, FGV Energia, 2017.

[4] FERNÁNDEZ-BLANCO, R. et al. Optimal energy storage siting and sizing: a wecc case study. IEEE Transactions on Sustainable Energy, IEEE, v. 8, n. 2, p. 733-743, 2016.

[5] SILVA, Y.; BORTONI, E. Sistemas de armazenamento de energia elétrica em redes inteligentes: Características, oportunidades e barreiras. Revista Brasileira de Energia, v. 22, p. $48-73,2016$.

[6] AGÊNCIA NACIONAL DE ENERGIA ELÉTRICA. Agência aprova 23 propostas da chamada de P\&D sobre armazenamento de energia. 2017. Disponível em: <http://www. aneel.gov.br/sala-de-imprensa-exibicao-2//asset_publisher/zXQREz8EVIZ6/content/ agencia-aprova-23-propostas-da-chamada-de-pd-sobre-armazenamento-de-energia/ 656877> . Acesso em: 13 de abril de 2019.

[7] CONDE, M. R. et al. O uso da avaliação de impactos no planejamento energético: escalas e abordagens adaptadas a diferentes níveis de decisão. Cigré, EPE, 2015.

[8] ZHAO, $\mathrm{H}$. et al. Review of energy storage system for wind power integration support.

Applied energy, Elsevier, v. 137, p. 545-553, 2015.

[9] VARGAS, L. S.; BUSTOS-TURU, G.; LARRAÍN, $F$. Wind power curtailment and energy storage in transmission congestion management considering power plants ramp rates.

IEEE Transactions on Power Systems, IEEE, v. 30, n. 5, p. 2498-2506, 2014.

[10] DENHOLM, P.; MAI, T. Timescales of energy storage needed for reducing renewable energy curtailment. Renewable energy, Elsevier, v. 130 , p. $388-399,2019$. 
[11] CARRIÓN, M.; DVORKIN, Y.; PANDŽIC, H. Primary frequency response in capacity expansion with energy storage. IEEE Transactions on Power Systems, IEEE, v. 33, n. 2, p. $1824-1835,2017$.

[12] COLE, W. J. et al. Utility-scale lithium-ion storage cost projections for use in capacity expansion models. IEEE, 2016.

[13] ASSOCIAÇÃO BRASILEIRA DE ENERGIA EÓLICA. Números ABEEólica: Fevereiro de 2019. 2019. Disponível em: <http://abeeolica.org.br/wpcontent/uploads/2019/02/NúmerosABEEólica-02.2019.pdf $>$. Acesso em: 8 de agosto de 2019.

[14] ELECTRIC POWER RESEARCH INSTITUTE. Energy Storage Integration Council

(ESIC) Energy Storage Request for Proposal Guide. Palo Alto, Califórnia, EUA, 2017.

[15] EMPRESA DE PESQUISA ENERGÉTICA. Estudos para a expansão da geração: Modelo de decisão de investimentos para expansão do SIN - Versão PDE 2027. Brasília, DF, 2018.

$34 \mathrm{p}$.

[16] TEIXEIRA, T. P. Modelagem da Utilização de Baterias em Sistemas Elétricos com Alta Inserção de Geração Eólica. 170 p. Dissertação (mestrado) - Universidade Federal do Rio de Janeiro - UFRJ/COPPE, 2018.

[17] ZAKERI, B.; SYRI, S. Electrical energy storage systems: A comparative life cycle cost analysis. Elsevier, Aalto, Finland, p. 28, out. 2014.

[18] NOVA. Em Busca da Superbateria. Produção: Daniel McCabe. 2017. 